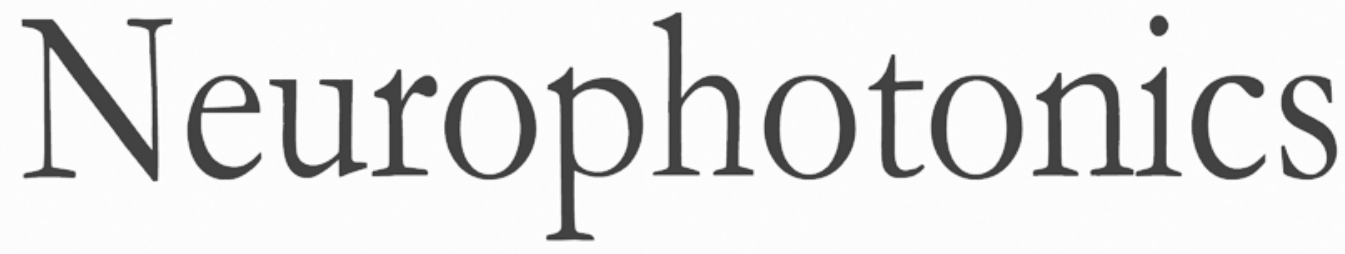

\title{
Targeted ischemic stroke induction and mesoscopic imaging assessment of blood flow and ischemic depolarization in awake mice
}

\author{
Matilde Balbi \\ Matthieu P. Vanni \\ Gergely Silasi \\ Yuki Sekino \\ Luis Bolanos \\ Jeffrey M. LeDue \\ Timothy H. Murphy
}




\title{
Targeted ischemic stroke induction and mesoscopic imaging assessment of blood flow and ischemic depolarization in awake mice
}

\author{
Matilde Balbi, ${ }^{a}$ Matthieu P. Vanni, ${ }^{a}$ Gergely Silasi, ${ }^{a}$ Yuki Sekino, ${ }^{\text {a }}$ Luis Bolanos, ${ }^{a}$ Jeffrey M. LeDue, ${ }^{\text {a,b }}$ and \\ Timothy H. Murphy ${ }^{a, b, *}$ \\ aUniversity of British Columbia, Department of Psychiatry, Kinsmen Laboratory of Neurological Research, Detwiller Pavillion, Vancouver, \\ British Columbia, Canada \\ bUniversity of British Columbia, Djavad Mowafaghian Center for Brain Health, Vancouver, British Columbia, Canada
}

\begin{abstract}
Despite advances in experimental stroke models, confounding factors such as anesthetics used during stroke induction remain. Furthermore, imaging of blood flow during stroke is not routinely done. We take advantage of in vivo bihemispheric transcranial windows for longitudinal mesoscopic imaging of cortical function to establish a protocol for focal ischemic stroke induction in target brain regions using photothrombosis in awake head-fixed mice. Our protocol does not require any surgical steps at the time of stroke induction or anesthetics during either head fixation or photoactivation. In addition, we performed laser speckle contrast imaging and widefield calcium imaging to reveal the effect of cortical spreading ischemic depolarization after stroke in both anesthetized and awake animals over a spatial scale encompassing both hemispheres. With our combined approach, we observed ischemic depolarizing waves ( 3 to $5 \mathrm{~mm} / \mathrm{min}$ ) propagating across the cortex 1 to $5 \mathrm{~min}$ after stroke induction in genetically encoded calcium indicator mice. Measures of blood flow by laser speckle were correlated with neurological impairment and lesion volume, suggesting a metric for reducing experimental variability. The ability to follow brain dynamics immediately after stroke as well as during recovery may provide a valuable guide to develop activity-dependent therapeutic interventions to be performed shortly after stroke induction. @2017 Society of Photo-Optical Instrumentation Engineers (SPIE) [DOI: 10.1117/1.NPh.4.3.035001]
\end{abstract}

Keywords: awake; calcium imaging; mesoscale; photothrombosis; spreading depolarization.

Paper 17024RR received Feb. 27, 2017; accepted for publication Jun. 16, 2017; published online Jul. 14, 2017.

\section{Introduction}

Stroke is the second leading cause of death and disability worldwide. ${ }^{1}$ Despite ongoing research and the enormous medical and economic burden on society ( $2 \%$ to $4 \%$ of total healthcare costs are attributed to stroke patients), ${ }^{2}$ limited therapeutic options are available for only a small portion of stroke patients in the acute phase. ${ }^{3}$ A large number of experimental therapies have shown beneficial effects in the laboratory, but few have reached the clinic. ${ }^{4}$ There are only a limited number in active development such as NA-1, the first drug in a class of neuroprotectants-PSD95 inhibitors-which successfully showed a reduction of stroke damage. ${ }^{5}$ These shortcomings emphasize the limitations of modeling human stroke in laboratory animals, where invasive surgical manipulations are part of most procedures. Despite the advances in developing experimental stroke models, confounding factors such as anesthetics used to ensure animal welfare during the induction of stroke have yet to be overcome. Common anesthetics, such as isoflurane and barbiturates, have been found to interfere with a series of signaling pathways, including GABAergic and glutamatergic, all of which are involved in the potential treatment of stroke. ${ }^{6,7}$ Moreover, isoflurane and other inhaled anesthetics can produce abnormal increases in cerebral blood flow. ${ }^{8}$

*Address all correspondence to: Timothy H. Murphy, E-mail: thmurphy@mail. ubc.ca
A recent study clearly shows that the presence of isoflurane anesthesia during stroke induction influences the effectiveness of neuroprotective drugs, ${ }^{9}$ highlighting the need for more refined methodologies when evaluating therapeutics.

Given the recent development of elegant in vivo preparations that provide optical access to the cortical surface in awake, headfixed mice ${ }^{10,11}$ we sought to validate a method for inducing targeted photothrombosis through the intact skull in awake mice. Taking advantage of the semitransparent nature of the mouse skull, transcranial windows are reliable preparations for longitudinal imaging and optical manipulation, providing a large, bihemispheric access to the cortex and reduced invasiveness. ${ }^{10}$ We recently described a chronic transcranial window preparation, previously used for imaging of both awake and anesthetized mice, which provides sufficient physical stability over a large field of view and can be used for longitudinal mesoscopic mapping of cortical function in awake mice. ${ }^{11,12}$ In this study, we make use of the optical access offered by this preparation and evaluate a protocol to optically induce focal ischemia to target-specific cortical areas ${ }^{13}$ using photothrombosis. ${ }^{14}$ Our protocol does not require any use of anesthetics either during head fixation or stroke induction. In addition, our approach combines laser speckle contrast imaging ${ }^{15}$ and wide-field calcium imaging to reveal brain dynamics and spreading peri-infarct depolarizations after stroke over a spatial scale

$2329-423 X / 2017 / \$ 25.00$ (c) 2017 SPIE 
encompassing both hemispheres, indicating that our preparation will facilitate functional cortical mesoscopic mapping during stroke and recovery.

\section{Material and Methods}

All procedures were approved by the University of British Columbia Animal Care Committee and conformed to the Canadian Council on Animal Care and Use guidelines and reported according to the animal research: reporting of in vivo experiments guidelines.

GCaMP3 mice were used to investigate spreading waves of activity after stroke. Data from negative GCaMP6 and ChR2 mice were pooled together for the laser speckle imaging, behavioral assessment, and histological quantification. Transgenic GCaMP3 mice of either sex $(n=16), 4$ to 5 months of age were produced by crossing homozygous B6.129S2-Emx1tm1 (cre)Krj/J strain (Jax No. 005628) with hemizygous B6; 129S-Gt(ROSA)26Sortm38(CAG-GCaMP3)Hze/J strain (Jax No. 014538, Ai38). ${ }^{12}$ Negative GCaMP6 and ChR2 mice were used for speckle imaging and behavior assays. The absence of one allele yielded the absence of expression of the light sensitive indicator, opsin. Transgenic negative GCaMP6 mice of either sex $(n=12), 4$ to 5 months of age were produced by crossing Emx1-cre B6.129S2-Emx1tm1(cre)Krj/J, (Jax No. 005628), CaMK2-tTA B6.Cg-Tg (Camk2a-tTA) 1Mmay/DboJ, (Jax No. 007004) and either TITL-GCaMP6s Ai94; B6.CgIgs7tm94.1(tetO-GCaMP6s)Hze/J, (Jax No. 024104), or TITL-GCaMP6f Ai93; B6.Cg Igs7tm93.1(tetO-GCaMP6f) Hze/J, (Jax No. 024103) strains. ${ }^{16}$ PCR confirmed the absence of either TITL-GCaMP6, CaMK2-tTA, or Emx1-cre sequence.

ChR2 mice of either sex $(n=12), 4$ to 5 months of age from the Jackson Laboratory, line 18, stock 007612, strain B6.Cg-Tg (Thy1-COP4/EYFP) 18Gfng/J were used as well.

\subsection{Chronic Transcranial Window Implant}

The window preparation was performed as previously described. ${ }^{11}$ Briefly, mice were anesthetized with isoflurane (2\% induction, $1.5 \%$ maintenance in air) and then mounted in a stereotactic frame. To maintain the corneal moisture, the eyes were covered with eye lubricant (Lacrilube; Ref. 17) and the body temperature was kept at $37^{\circ} \mathrm{C}$ using a feedbackregulated heating pad monitored by a rectal probe. Mice received an injection of lidocaine $(0.1 \mathrm{ml}, 0.2 \%)$ under the scalp and a subcutaneous injection of saline solution containing burprenorphine $(2 \mathrm{mg} / \mathrm{ml})$, atropine $(3 \mu \mathrm{m} / \mathrm{ml})$, and glucose $(20 \mathrm{mM})$. The skin between the ears and the eyes was shaved and properly cleaned with betadine dissolved in water and ethanol.

The skin covering the occipital, parietal, and frontal bones was cut away. The fascia and any connective tissue on the skull surface were gently wiped off. C\&B Metabond transparent dental cement (Parkell, Edgewood, New York; Product: C\&B Metabond) was prepared in a ceramic bowl and used to glue a head-fixing screw to the cerebellar plate. With the setscrew in place, a thick layer of dental adhesive was applied on the skull. A coverglass (Marienfeld, Lauda-Konigshofen, Germany; Cat. No.: 0111520) previously cut to the size of the final cranial window $(11 \mathrm{~mm} \times 9 \mathrm{~mm})$ was placed on top of the dental cement before it solidified, preventing the formation of bubbles. The cement remains transparent after it solidifies, and the surface vasculature should be readily seen through the final result.

\subsection{Recovery and Postoperative Monitoring}

At the end of the procedure, mice received a second subcutaneous injection of saline $(0.5 \mathrm{ml})$ with $20 \mathrm{mM}$ of glucose and were allowed to recover in their home cages with an over-head heat lamp. Activity level of mice that underwent the procedure was monitored hourly for the first $4 \mathrm{~h}$ and every 4 to $8 \mathrm{~h}$ thereafter. Mice are allowed to recover for 7 days from the window implantation before performing baseline measurements.

\subsection{Setup and Procedure to Induce Stroke}

Mice were head-fixed using a slightly modified version of an apparatus previously described for imaging awake mice. ${ }^{11} \mathrm{~A}$ locking ball and socket mount (Thorlabs, Product: TRB1) was mounted on the platform where the stroke was induced using two optical posts and a 90-deg turn. The head-fixing screw (4/40 threads) was attached to the ball and socket mount (8/32 threads) using a thread adapter (AP84E, Thorlabs). A clear Plexiglas tube (28-mm diameter) was attached on the same platform to provide a chamber for the mouse. The setscrew was fitted with a nut and threaded into the ER025 post by rotating the ball within the socket. The nut was subsequently tightened upward against the ER205 post to immobilize the head without applying pressure to the cranial window. After a period of habituation, when mice are introduced to the apparatus and head-fixed daily for a few minutes, mice can be gently persuaded into the tube. Once habituated, mice show minimal signs of struggling and can show normal behaviors, such as whisking or grooming.

A target area between sensory and motor cortex-stereotactic coordinates $(1.5 ; 0.5) \mathrm{mm}$ from bregma-was previously identified based on response during intrinsic optical signal imaging. ${ }^{18}$

To induce photothrombotic occlusion, ${ }^{14}$ we used a custom-made compact laser system ${ }^{13}$ (Fig. 1) consisting of a 40-mW diode pump solid-state 532-nm laser MGM-20 (Beta Electronics, Columbus, Ohio) attenuated to $11 \mathrm{~mW}$ at the window surface through a polarizer. Beam diameter is $1.2 \mathrm{~mm}$ and it was measured at full width at half max amplitude. The system includes a fan and heat sink as well as a transistor-transistor logic-triggered power supply. While wearing safety goggles, the laser was turned on and targeted to the preselected area within the cranial window using bregma as a landmark. Once the laser was correctly targeted, the laser was turned off, and all the clamps were tightened to prevent movement. Previous reports showed that this amount and duration of the green laser irradiation did not produce tissue damage or impact the targeted vessels in any way unless combined with Rose Bengal (RB) administration. ${ }^{20,21}$

Using an insulin syringe, mice were injected intraperitoneally (IP) ( $0.1 \mathrm{cc}$ per $10 \mathrm{~g}$ of body weight) with a photosensitive dye solution-RB (R3877-5G, Sigma-Aldrich, St. Louis, Missouri). The solution was freshly prepared by dissolving $0.01 \mathrm{~g}$ of $\mathrm{RB}$ in $1 \mathrm{ml}$ of saline solution. The mix was vortexed (Fischer Vortex, Fischer Scientific, Hampton, New Hampshire) and sonicated (Branson, Danbury, Connecticut). Exposure to light was avoided, and the solution was filtered with a $0.2-\mu \mathrm{m}$ filter (PALL Life Science, PN4612). RB was injected IP by gently holding the tail of the mouse and lifting it up to get a clear view of the abdomen. After the injection, we allow $2 \mathrm{~min}$ for the dye to reach the brain and then the laser illumination was turned on for $13 \mathrm{~min}$ at the targeted area to induce focal ischemia 

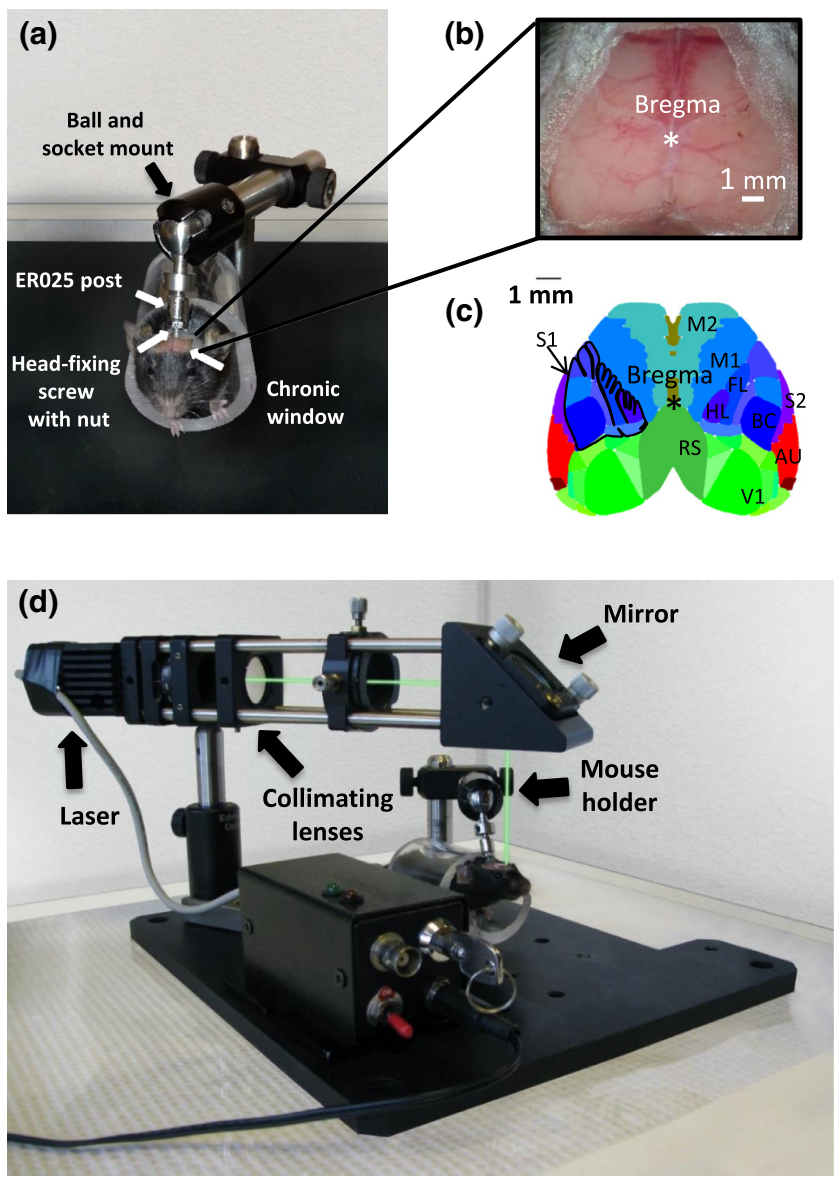

Fig. 1 Hardware for awake head-fixation and stroke induction. (a) A set screw was surgically implanted behind the cranial window to stably head fix the mouse. (b) Blow up of the chronic window preparation where surface vessels are visible through the intact skull, scale bar of $1 \mathrm{~mm}$. (c) Mouse brain atlas image (from the Allen Institute Atlas) ${ }^{19}$ showing cortical areas that may be targeted with this method. M1 and $\mathrm{M} 2$, primary and secondary motor areas, respectively; BC, barrel field; FL, forelimb; and HL, hindlimb regions of the primary somatosensory area, respectively (S1); S2, supplemental or secondary somatosensory area; $\mathrm{V} 1$, primary visual area; $\mathrm{AU}$, primary auditory area; and RS, retrosplenial area. (d) Mice are persuaded into a chamber mounted on the system for the stroke induction, and head-fixed while awake.

(Fig. 2). During stroke induction, the room lights were dimmed to increase comfort and reduce stimulation for the mouse. Sham surgery controls were treated in the same way with the omission of the laser illumination. In this case, the surgery did not produce any sign of damage. Due to the minimally invasive nature of the procedure, awake mice were imaged immediately after surgery.

\subsection{Laser Speckle Contrast Imaging}

Speckle imaging is based on blurring of interference patterns of scattered laser light by the flow of blood cells. ${ }^{15}$ However, the relationship of laser speckle signals with perfusion is not sufficiently robust to allow formal calibration and quantification in chronic repeated imaging sessions $;{ }^{22}$ for this reason, we present relative changes in blood flow and not absolute levels. To periodically assess blood flow, laser speckle imaging was performed before, immediately after stroke induction, and every other day during the first week after stroke in awake head-fixed mice. The

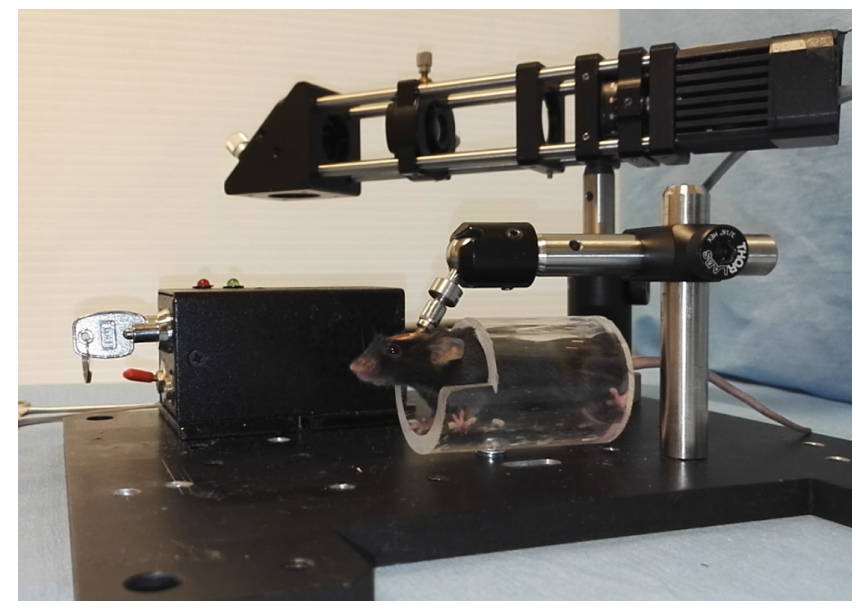

Fig. 2 Head fixation and stroke induction. Example of head fixation procedure of an awake mouse following that an area chosen on stereotactic coordinates is targeted for stroke induction. After an IP injection of RB (not shown), the laser is turned on for $13 \mathrm{~min}$ (Video 1, MOV, 473 KB [URL: http://dx.doi.org/10.1117/1.NPH.4.3 .035001.1]).

surface of the cortex was illuminated with a 784-nm 32-mW StockerYale SNF-XXX_785S-35 laser (Stocker\&Yale, Salem, New Hampshire) at 30-deg angle with a beam expander and light intensity controlled by a polarizer. Blood flow was detected by a CCD camera (Dalsa 1M60), and the image acquisition was performed using EPIX XCAP software (v3.7). Three hundred frames were acquired at $10 \mathrm{~Hz}$ with $10-\mathrm{ms}$ exposure time. For the assessment of speckle contrast over time, regions of interest (ROIs) were selected and centered at the site where the laser was targeted on the cortex.

Speckle contrast ( $k=$ standard deviation/mean intensity) was calculated using ImageJ software (National Institute Mental Health, Bethesda, Maryland). ${ }^{13}$ Blood flow measurements were scaled to the minimum flow rate that was determined from contrast values measured from the mouse cortex shortly after it was sacrificed by injection of pentobarbital sodium (Euthanyl, Bimeda-MTC, Cambridge, Ontario).

\subsection{Calcium Imaging Recordings}

Spreading depolarization (SD) is an acute hallmark of stroke. $^{23,24}$ These waves have been previously recorded using intrinsic signal imaging ${ }^{25}$ or electroencephalogram in anesthetized mice. ${ }^{26}$ To investigate functional cortical dynamics, fluorescent signals from awake GCaMP3 mice were recorded within 1 to 4 min following stroke. For comparison, another group was imaged under anesthesia using isoflurane (4\% induction, $1.2 \%$ maintenance in air). In anesthetized mice, body temperature was monitored and kept at $37^{\circ} \mathrm{C}$ using a feedbackregulated heating pad monitored by a rectal probe. Images of the cortical surface were recorded through a pair of back-toback photographic lenses (Nikon NIKKOR $f=50$ and $35 \mathrm{~mm}$ ) coupled to a $1 \mathrm{M} 60$ Pantera CCD camera (Dalsa). To visualize the cortex and vasculature, the surface of the brain was illuminated with green light. GCaMP3 was excited with blue-lightemitting diodes (Luxeon, $470 \mathrm{~nm}$ ) with bandpass filters (467 to $499 \mathrm{~nm}$ ). Emission fluorescence was filtered using a 510to 550-nm bandpass filter. 12-bit images were collected at 33.3- or 100-ms intervals, i.e., 10 to $30 \mathrm{~Hz}$ using XCAP imaging software. 


\subsection{Image Processing of Calcium Signals}

All data were imported and analyzed with MATLAB ${ }^{\circledR}$ (Mathworks, Inc., Natick, Massachusetts). The variations in fluorescence were calculated for each pixel by measuring the relative change between each frame and the average fluorescence during the first second of recording $(\Delta F / F o)$. The absolute time to peak was evaluated for each pixel by measuring the time to the maximum change of fluorescence. The relative time to peak was calculated by subtracting each absolute timeto-peak value by the minimum value within the hemisphere (initiation spot). The propagation speed was calculated by dividing the distance from each pixel of the hemisphere to the initiation pixel (in $\mathrm{mm}$ ) by the relative time-to-peak value (in $\mathrm{min}$ ).

\subsection{Neurodeficit Score Evaluation}

The neurodeficit score (NDS) is a standardized qualitative assessment for measuring the degree of motor impairment presented after stroke. ${ }^{27}$ The test was performed before stroke or sham induction to establish a baseline and then at day 1, 3, 5 , and 7 after the procedure. The mouse was placed on a flat surface and gently lifted by the base of the tail only until the forepaws were no longer touching the surface. The first criterion evaluates forelimb (FL) flexion, graded from 0 , where the mouse showed a uniform extension of the FL, to 2, where the mouse showed shoulder adduction with FL flexion (the wrist bent up toward the body). Similarly, hindlimb (HL) flexion was scored with 0 when the mouse stretched its HL caudally, parallel to its body, and 1 in the presence of hind limb flexion. Subsequently, the mouse was gently lifted by its tail and held about $15 \mathrm{~cm}$ above the flat surface. In this case, it was assigned a score of 0 if the mouse did not show a C-shaped bending of the body toward one side, 1 if the impairment was slightly present and 2 if the bending was severe. To evaluate forepaw grasping, the mouse was lifted by its tail and had to grasp a horizontal metal wire of a diameter of $2 \mathrm{~mm}$ with the FLs. A score of 0 was given if the mouse would grasp completely with both paws, and all digits wrapped around the bar, 1 if the grasp was normal with one paw but the mouse used some contralateral digits. A score of 2 was assigned if the mouse would grasp normally with one paw but then used contralateral wrist or arm, 3 if the mouse was unable to grasp with either paw. Before concluding the testing procedure, the mouse was placed on a surface and the HLs were gently displaced laterally of 1 to $2 \mathrm{~cm}$. A score of 0 was given if the mouse would immediately replace it, 1 if the replacement would occur within 0 to $20 \mathrm{~s}, 2$ within 20 s to $1 \mathrm{~min}$, and 3 if the replacement would occur in more than $1 \mathrm{~min}$ or not at all. After the testing, the mouse was placed back in his home cage until the next evaluation.

\subsection{Infarct Volume Analysis}

Mice were allowed to survive for 7 days. On day 7 , mice were perfused with phosphate-buffered saline and $4 \%$ paraformaldehyde, and the brains were harvested. To evaluate infarct volume, brains were sectioned at $50 \mu \mathrm{m}$ on a Leica vibratome in the coronal plane. Every third section (150- $\mu \mathrm{m}$ apart) was stained with $0.001 \%$ Fluoro Jade C (FJC). Wide-field images of FJC-stained sections were collected with a Zeiss AxioZoom Macroscope. Using ImageJ software (National Institute Mental Health, Bethesda, Maryland), the infarct area was outlined in each image. Infarct volume was estimated by adding the infarct area for each section multiplied by the distance between each section, which was $150 \mu \mathrm{m}$.

\subsection{Statistical Analysis}

To assess the effects of stroke on blood flow in the experimental groups, pairwise two-way analysis of variance (ANOVA) with the Bonferroni correction for posthoc comparisons was performed using a standard statistical software package, Prism 5 (GraphPad Software, La Jolla, California). The differences between baseline and day 1 as well as in infarct volume between groups were evaluated using the Mann-Whitney test. The differences between calcium signals were evaluated using the unpaired $t$-test. The groups of mice exhibiting SDs were compared using the Pearson's chi-squared test. Scheirer-Ray-Hare extension of the Kruskal-Wallis test was performed on the ordinal NDS data. Pearson's $r$ correlation coefficient was calculated between each daily measurement of NDS and laser speckle imaging and the postmortem lesion volume, as well as in pairwise comparisons between NDS and laser speckle imaging measurements. Results from blood flow measurements are shown as mean \pm SD. Infarct volumes are shown as median $\pm 75 / 25$ percentile. A value of $P<0.05$ was considered to be statistically significant.

\subsection{Randomization and Blinding}

All mice were randomly assigned to the procedures making sure of a balanced distribution between male and females in each group; a researcher blinded toward sham or stroke group assignment performed the surgical preparation and data analysis.

\section{Results}

Cranial windows were implanted during a surgical procedure at least 7 days prior to stroke induction as in Ref. 11. The procedure was simple to perform, requiring less than 30 min per mouse, which minimizes the exposure of the mouse to anesthesia. It resulted in a stable cranial window [Fig. 1(b)] with a large field of view of the cortex [Fig. 1(c)] with little disturbance to mouse behavior (grooming, feeding, and climbing). During habituation to head-fixing sessions, the setscrew kept the cranial window and entire head of the mouse firmly in place regardless of body movement [Figs. 1(a) and 1(d)]. ${ }^{11}$

This transcranial window preparation permitted the tracking of cerebral blood flow changes using laser speckle imaging [Fig. 3(a)]. ${ }^{15}$ Prior to stroke induction, baseline measurements were recorded clearly and without apparent optical distortion from the window. The head-fixing setscrew of the cranial window allowed us to perform the photothrombotic stroke in awake mice without major disturbances to the mouse or further need of invasive manipulation [Fig. 1(d)]. Laser exposure in mice injected with RB resulted in the formation of thrombus within 13 min similar to the time required using other existing window preparations in anesthetized animals. ${ }^{28}$ Mice remained awake during the procedure and did not exhibit signs of pain or discomfort, such as audible vocalizations or writhing. Following the procedure, mice are monitored for the first few hours and daily thereafter; mice do not show overt signs of distress, such as hunching over, shaking, or aggression. Given the lack of aggressive behavior, we continue to group house mice after the awake stroke procedure.

On day 1 (24 h after stroke), blood flow within the targeted area was examined using laser speckle imaging 
(a)

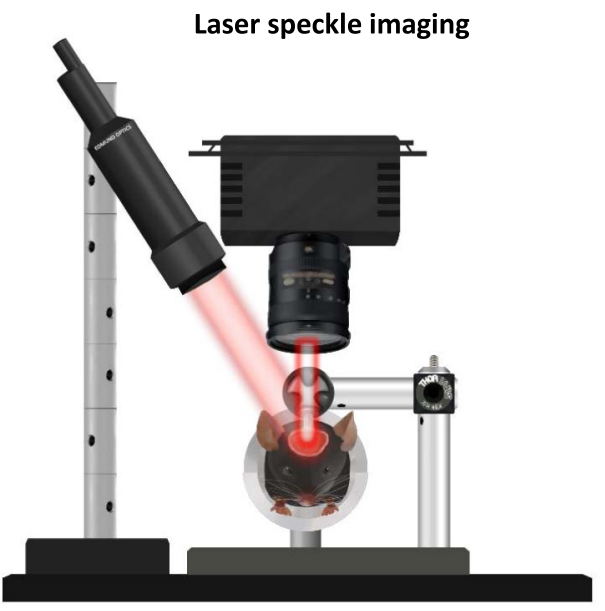

(c)

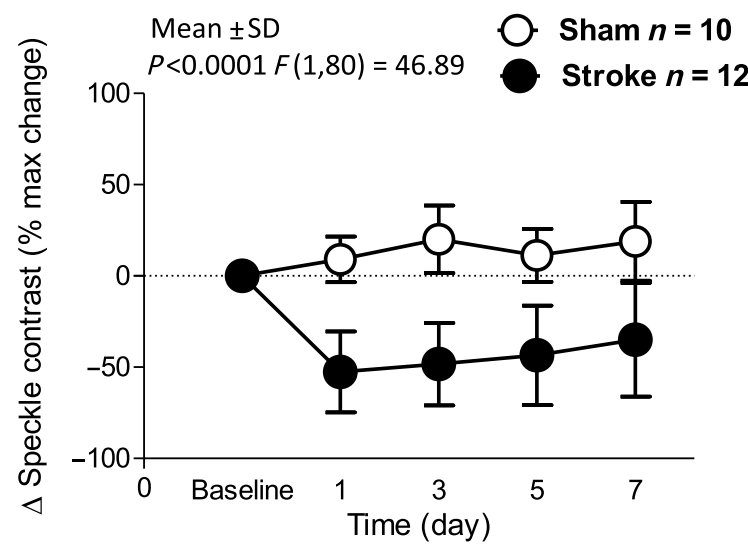

(b)

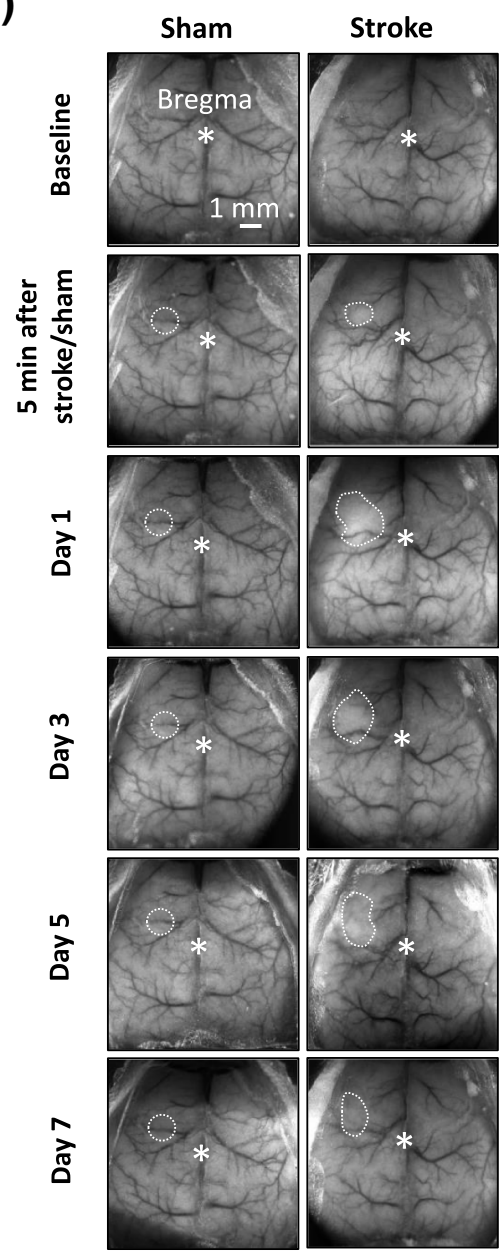

Fig. 3 Laser speckle imaging for changes in blood flow following ischemic stroke. (a) Drawing of the laser speckle imaging setup where the head-fixed mouse is placed while fully awake. The imaging is performed in a darkened room without visual, tactile, or auditory stimuli. (b) Representative pictures of laser speckle contrast imaging of cortical blood flow at baseline and at $5 \mathrm{~min}, 1,3,5$, and 7 days following sham or stroke surgery. Dotted lines (drawn by hand) are used to highlight the infarcted area. Scale bar of $1 \mathrm{~mm}$. (c) Quantification of the changes in cortical blood flow expressed in percentage of baseline in mice that underwent sham (white symbols $n=10$ ) and stroke surgery (black symbols $n=12$ ). Pairwise twoway ANOVA with the Bonferroni correction for posthoc comparisons. Mean $\pm \mathrm{SD}$.

[Figs. 3(a)-3(c)]. Blood flow was significantly lower in stroke mice within the targeted area (ROI included the area between sensory and motor cortex, where the stroke was induced) $(P<0.0001)$. The results from the two-way ANOVA on the complete time course, i.e., until day 7 , corroborate the significance of the effect of stroke performed in awake mice on local blood flow [Fig. 3(c); $P<0.0001, F_{(1,80)}=46.89$ ]. Stroke was induced in the area between sensory and motor cortex (stereotactic coordinates $1.5-\mathrm{mm}$ lateral and $0.5-\mathrm{mm}$ anterior of bregma) in awake and anesthetized mice, and functional investigation was performed in parallel. Using wide-field calcium imaging within $5 \mathrm{~min}$ from the stroke induction [Figs. 4(a)-4(h)], ischemic depolarizing waves were observed in 6 of the 10 awake mice and 2 of the 4 anesthetized mice (chi-squared test, $P=0.732$ ) (Fig. 5). Observed waves, one for each mouse during the imaging session, had a significantly smaller magnitude of calcium change from resting baseline in awake mice, (maximum $\Delta F / F: 97 \% \pm 13 \%$ versus $223 \% \pm 22 \%$ for awake and anesthetized mice, respectively, $P=0.002$ ).
Median propagation speed $(5.16 \pm 0.48$ versus $3.96 \pm$ $0.84 \mathrm{~mm} / \mathrm{min}, \quad P=0.269)$ was not significantly different between awake and anesthetized mice, and no relationship was observed between speed and amplitude [Pearson's correlation: $r=-0.29, P=0.48$, Fig. 4(i)]. Propagation speed was in the same range of previous findings ( 3 to $5 \mathrm{~mm} / \mathrm{min}$ ), where depolarizing waves were investigated in anesthetized mice and rats using electrophysiological recordings and optical intrinsic signal imaging. ${ }^{29,30}$ No propagating wave of that order of magnitude was observed in the opposite hemisphere or in mice that underwent the sham procedure (not shown). Mice were subsequently returned to their home cages.

Both stroke and sham mice received an NDS ${ }^{27}$ of 0 during baseline assessment. Stroke mice received a score significantly different from baseline $(P<0.0001)$ and the sham mice $\left(P<0.0001, F_{(4,88)}=31.62\right)$, which never received an NDS other than 0 [Fig. 6(a)].

On day 7, mice were euthanized, and the infarct volume was quantified. The infarct area was outlined with FJC 
(a) Awake photothrombosis

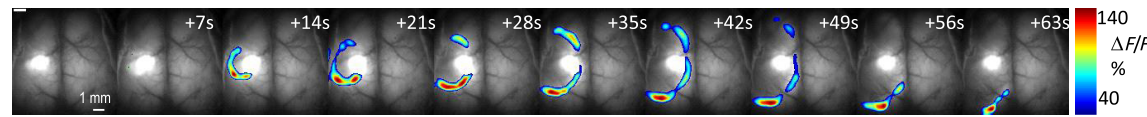

(b)

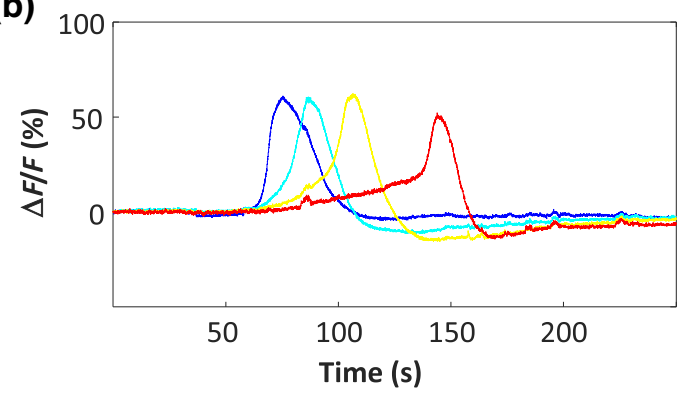

(c)

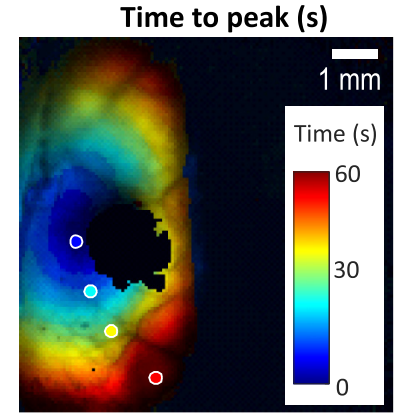

Anesthetized photothrombosis

(d)

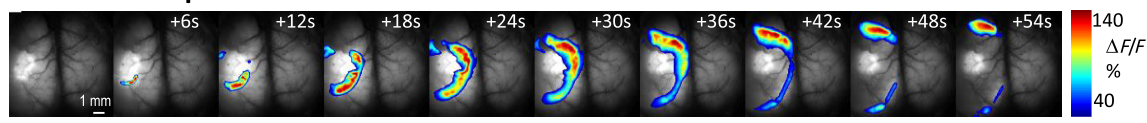

(e)

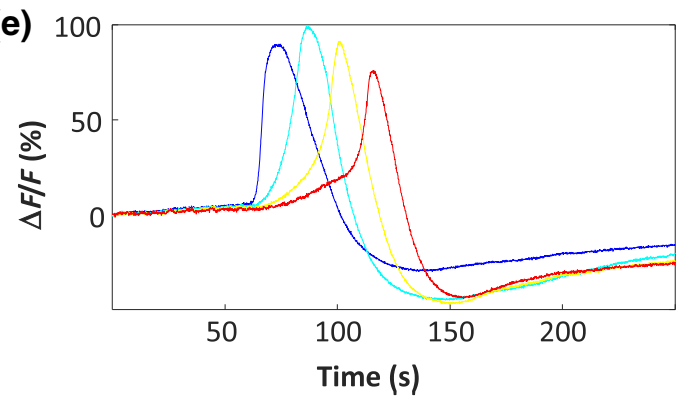

(g)
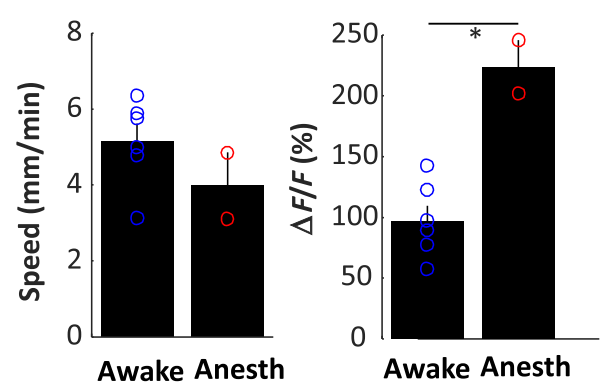

Time to peak (s)

(f)

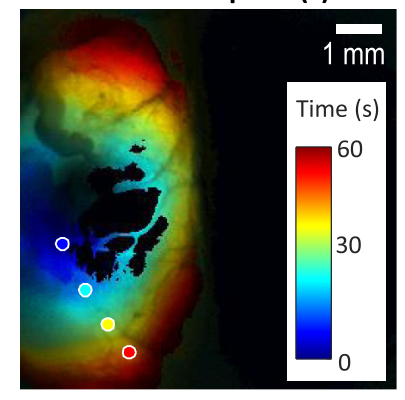

(h)

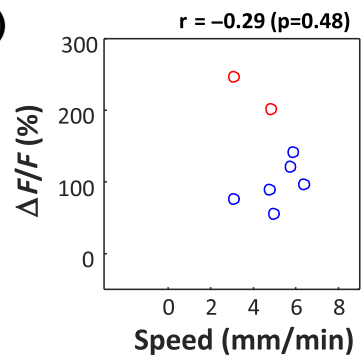

Fig. 4 Calcium signaling imaging in awake and anesthetized mice following ischemic stroke. (a) Montage of the raw fluorescence recorded at 7-s interval in an awake mouse. (b) Fluorescence recorded in four different points indicated in (c). (c) Estimation of the relative time to peak of the propagation wave (color) normalized by the amplitude $(\Delta F / F o$, luminance). (d) Montage of the raw fluorescence recorded at 6-s interval in an anesthetized mouse. (e) Fluorescence recorded in four different points indicated in (f). (f) Estimation of the relative time to peak of the propagation wave (color) normalized by the amplitude $(\Delta F / F o$, luminance). Black pixels represent those saturated at any point during the recording. (g) Distribution of the wave speeds and amplitude as well as (h) relationship between them (scatter plot) in awake and anesthetized mice. Chi-squared test was used to assess differences between the two groups.

staining [Fig. 6(b)]. This type of damaged tissue was visible in the brain slices from all mice that received the stroke procedure, resulting in a median lesion volume of $0.9 \pm$ $0.811 \mathrm{~mm}^{3}$, with a minimum volume of $0.2 \mathrm{~mm}^{3}$ and a maximum of $2.8 \mathrm{~mm}^{3}$, in contrast to mice that received the sham procedure, which had a lesion volume of $0 \mathrm{~mm}^{3}$, a statistically significant difference between procedures $[P=0.0005$; Fig. 6(c)].
Lesion volume measured at day 7 was inversely correlated with changes in blood flow on each day after stroke induction, confirming the association between the decrease in blood flow and the infarcted area [Figs. 6(d) and 6(g)]. Lesion volume was positively correlated with NDS on days $1(r=0.685$, $P=0.014)$ [Fig. 6(e)] and $3(r=0.7272, P=0.0074)$, but not at day 5 and 7 [Fig. 6(g)]. A negative correlation was also found between NDS and changes in blood flow on days 1 


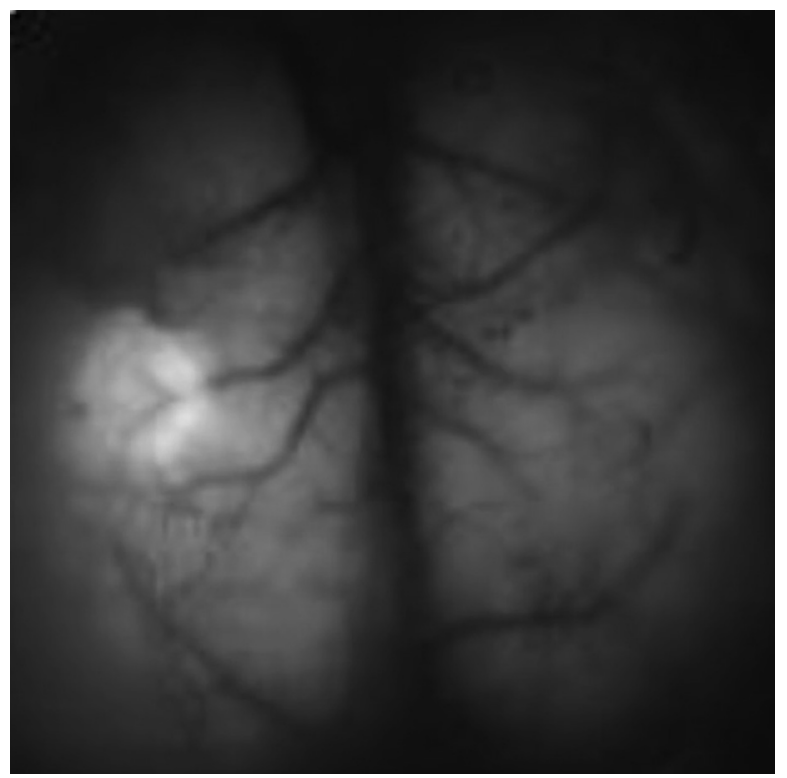

Fig. 5 SD in targeted stroke. Calcium signaling imaging in anesthetized and awake mice following ischemic stroke shows spreading depolarizing waves a few minutes after stroke induction. Speed $45 \times$ (Video 2, MP4, 10,105 KB [URL: http://dx.doi.org/10.1117/1.NPH.4.3 $.035001 .2])$.

$(r=-0.6158, P=0.033)$ [Fig. 6(f)] and $3(r=-0.5763$, $P=0.0499)$ [Fig. 6(g)].

\section{Discussion}

We developed a method for stroke induction in awake, headfixed mice avoiding potential confounds from anesthesia. This method is combined with longitudinal mesoscopic mapping of cortical function through the optical access facilitated by a transcranial window preparation.

To induce focal ischemic stroke in awake mice, we used the photothrombotic model initially proposed by Rosenblum and El-Sabban, ${ }^{31}$ and improved upon by Watson et al. ${ }^{14}$ The method is based on the light-dependent production of reactive oxygen species and triggering of clotting cascades after an injected photosensitive dye, such as RB, is irradiated. ${ }^{14}$

In a limited number of previous studies, invasive approaches such as the implantation of brain cannulas to deliver endothelin ${ }^{32}$ were used in awake animals to induce stroke. Only recently, Seto et al. ${ }^{9}$ developed a protocol for inducing focal ischemic stroke in awake mice inducing photothrombosis in a way that significantly reduces invasive manipulation. In this protocol, laser light needed for photothrombosis was focused on a plastic optical fiber, which requires the installation of a headcap on the skull of the mouse. Despite the procedure being less invasive than previously described, the fiber stroke protocol still required 15 to $20 \mathrm{~min}$ of prior exposure to anesthesia to thin the skull and attach the headcap. ${ }^{9}$ The thinning skull procedure itself may be a source of variability between mice and can mechanically disrupt the underlying structures with consequent microglia and astrocyte activation..$^{33}$ A nonuniform skull thickness may cause spherical aberrations and distortion of fluorescent structures located deep in the cortex. ${ }^{34}$ Moreover, mice were tethered to the optical fiber for the time of occlusion $\sim 60 \mathrm{~min}$, which may limit natural behaviors of mice or induce a stress response. ${ }^{9}$ The placement of a fiber cannula also obscures the measurement of functional signals such as blood flow that can be important to define the extent of lesions for later recovery enhancing treatments. With our approach using a head-fixing screw ${ }^{11}$ (that facilitates the immobilization of mice while preserving optical access), we eliminate anesthesia at stroke induction and assessment. Anesthesia has long lasting effects on brain function and it can reduce the extent of ischemic damage and mask the beneficial outcome of a potential therapy. ${ }^{9}$ Another advantage of a protocol that does not require anesthesia is that it allows chronic daily imaging, which would otherwise increase the exposure to confounding factors in the form of repeated anesthetic administration for either stroke induction or imaging assessments. There is evidence that the effects of anesthetics may have prolonged effects in mice. ${ }^{35}$ Moreover, most anesthetics depress myocardial contractility, arterial blood pressure, and are often respiratory depressants. ${ }^{36,37}$ Daily exposure to anesthetics alters systemic physiology, and its effects outlast the duration of anesthesia. In our protocol, stroke induction is a relatively less invasive procedure and can be easily targeted to a specific cortical region, such as the FL representation as identified during sensory mapping or estimated for stereotaxic coordinates. This is an advantage for the study of specific cortical regions targeted by stroke, as well as regions that are functionally connected to the damaged area and the peri-infarct cortex. ${ }^{38}$ However, the lesion target was defined based on stereotactic coordinates, without doing any discrimination based on type or dimensions of surface vessels. Variability in vessel distribution of each mouse could explain a range of lesion volumes, ${ }^{21}$ although the photoactivated area is large $\sim 1 \mathrm{~mm}$ in diameter and should contain all classes of surface vessels. Future refinements to the procedure could target individual identified arterioles for photothrombosis ${ }^{39,40}$ or employ magnetic nanoparticles for higher precision. ${ }^{41}$ Notably, our analysis suggests that changes in blood flow are a good predictor for variability in lesion volume providing a method to potentially stratify the animals in future work where lower variability within groups is desired.

Existing methods for brain imaging in awake head restraint animals employ strategies to reduce the stress response during imaging. An example of this approach is the use of an air-supported spherical treadmill to allow walking and running. ${ }^{42}$ However, our system uses a relatively simpler approach to both induce stroke and image the brain after the mouse is habituated to the head fixation.

In this context, our preparation uses a chronic window that allows us to perform bihemispheric wide-field imaging in awake mice immediately after receiving stroke. This preparation is quick to perform with little or no risk of damage or inflammation that can easily occur with more invasive techniques such as bone thinning. ${ }^{33}$ More relevant to our purposes is the investigation of events that happen over a larger spatial scale after stroke. The wide field of view of the window facilitates the investigation of multiple ROIs and correlation with functional maps. We have shown that with our combined approach we could reveal brain dynamics after stroke at a mesoscopic level. Using wide-field calcium imaging, we could observe ischemic depolarizing waves in awake mice a few minutes after stroke induction. These depolarizing waves have been previously imaged in anesthetized mice, ${ }^{23,43}$ however, our data are the first to show that ischemic waves propagate across the cortex in awake mice. The effects of anesthetics on ischemic wave propagation can involve a variety of mechanisms, such as reduced energy depletion as a result of suppression of electrical activity, interference with pathophysiologic processes such as disturbance of 
(a)

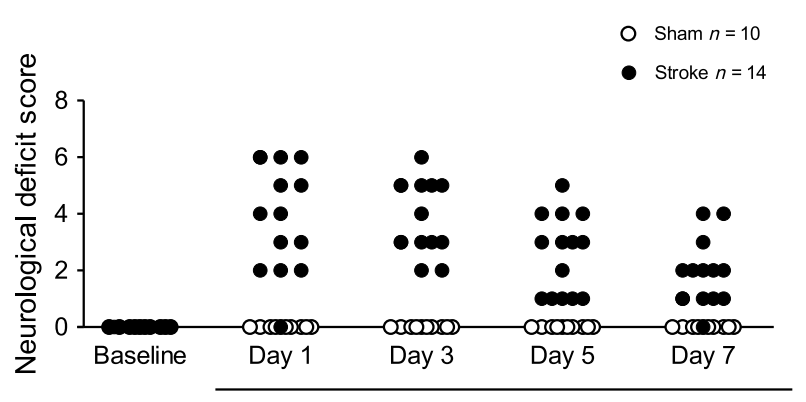

Post-stroke time

(d)

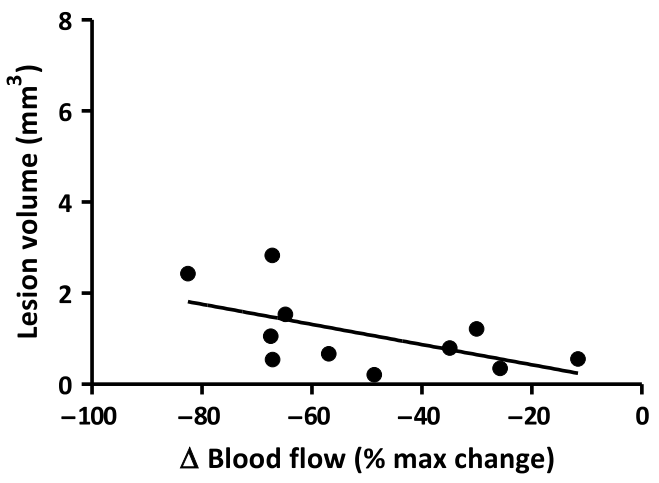

(f)

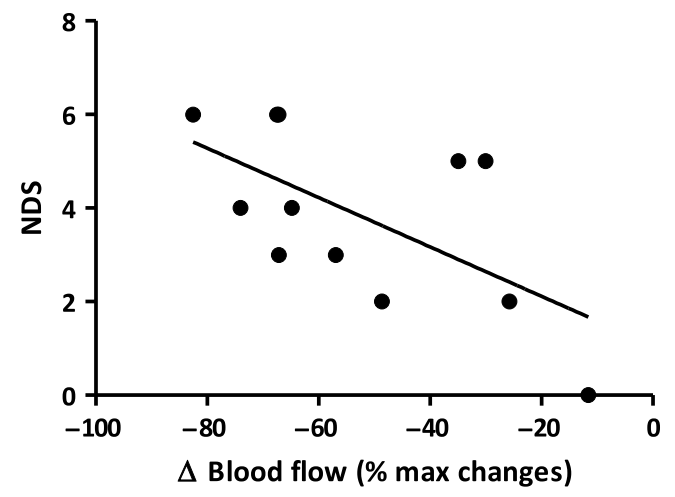

(b)

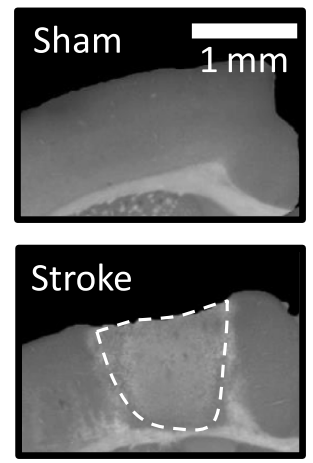

(c)

Median $\pm 75 / 25$ percentile

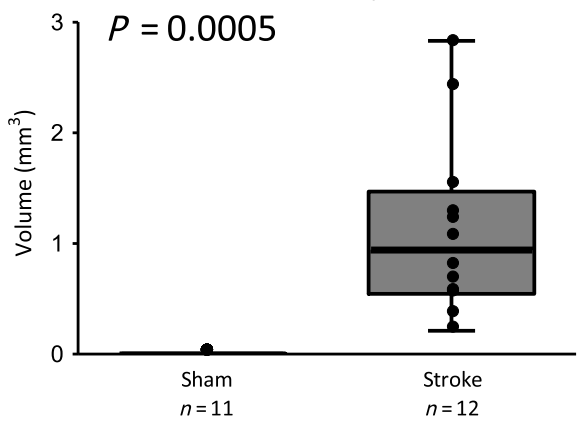

(e)
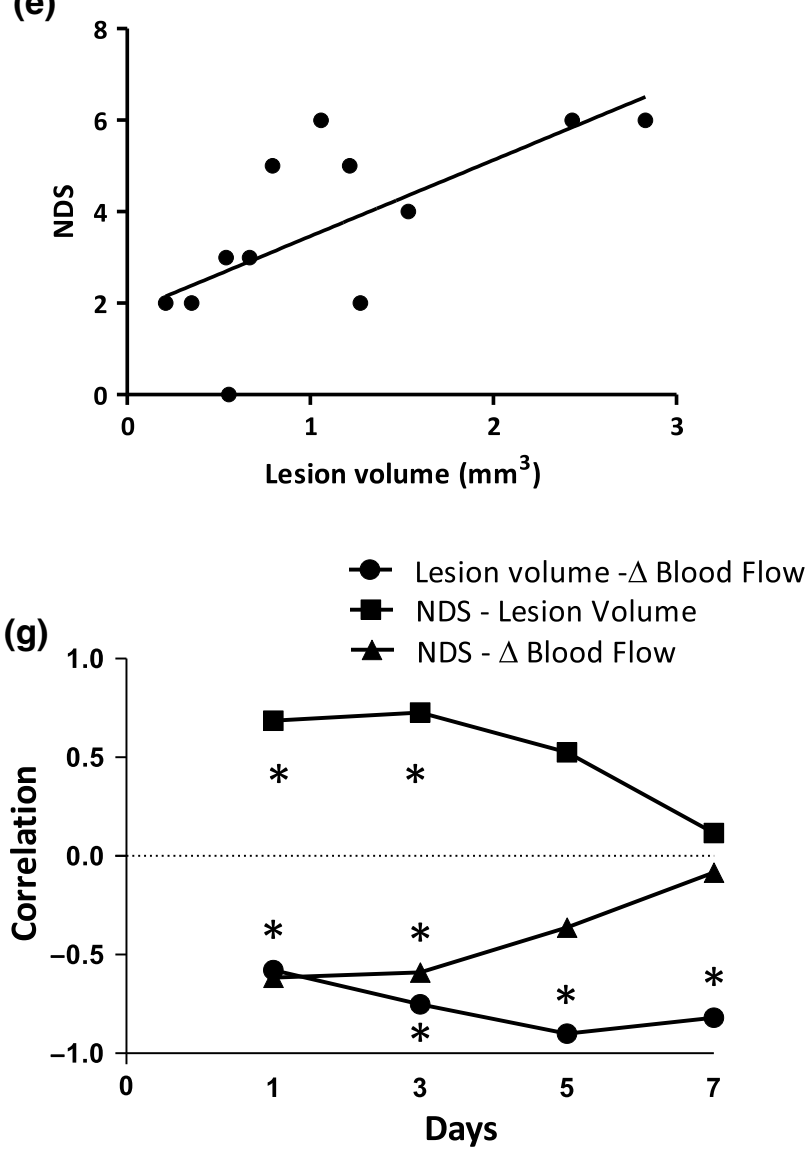

Fig. 6 Neurological deficit score and histological quantification following ischemic stroke. (a) NDS assessed in sham (white symbols $n=10$ ) and stroke (black symbols $n=14$ ) mice. (b) Representative pictures showing ROls (cortex) of coronal brain sections stained with FJC for ischemic cell death. Scale bar of $1 \mathrm{~mm}$. (c) Quantitative analysis of infarct volume 7 days after sham $(n=11)$ or stroke $(n=12)$ surgery. Correlation at day 1 between: (d) lesion volume and changes in blood, (e) lesion volume and NDS, (f) changes in blood flow and NDS one day following stroke induction. (g) Time courses of correlation between lesion volume and changes in blood flow, NDS and lesion volume, and NDS and changes in blood flow. Results from NDSs were evaluated with the Scheirer-Ray-Hare extension of the KruskalWallis test. Infarct volume between groups was evaluated using the Mann-Whitney test, and data are shown as median $\pm 75 / 25$ percentile. Pearson's $r$ correlation coefficient was used to assess correlations.

ion homeostasis, or accumulation of excitotoxins during ischemia and postischemic free radical formation. All of which could potentially interfere with the wave's propagation. Among those factors, intracellular communication through gap junctions should be taken into account considering that commonly used anesthetics such as halothane are gap junction blockers. ${ }^{44}$ Furthermore, the ability to visualize mesoscopic cortical activity may prove valuable in understanding critical neurovascular processes at the acute stage after stroke. One limitation of our approach is the inability to perform GCaMP 
functional imaging at early time points following stroke induction within the peri-infarct cortex. This region becomes excessively bright due to apparently high intracellular calcium masking any smaller functional signal. Moreover, the low number of mice used to compare wave propagation in anesthetized and awake mice does not provide an exhaustive comparison and should be considered preliminary. The aim of our study was to develop a protocol that dispenses with the used anesthetics and allows functional investigations in short time.

Low mortality, the ability to target the infarct within different functional subdivisions of the cortex, and the minimal surgical intervention required are all advantages of this photothrombotic stroke model. The relatively small size of the infarct allows this model to more faithfully mimic the usual cases of human stroke, where most strokes are small in size and associated with some degree of recovery. ${ }^{45}$ Stroke models that require suture occlusion of the middle cerebral artery frequently produce tissue damage in a substantial portion of the ipsilateral hemisphere. ${ }^{46}$ Moreover, the reproducibility of infarction is affected by invasive procedural factors such as suture diameter that have been shown to correlate with the infarct volume. ${ }^{47}$ We suggest that parallel monitoring of regional blood flow may be used to further sort animal groups based on the degree and location of ischemia. It is particularly important to control these metrics in studies seeking to promote circuit level plasticity and recovery. However, it must be considered that this is a regionalrelative measure that may not reflect the full loss of blood within the infarcted core.

A stroke model that does not require anesthesia permits both the assessment and alteration of activity at early time points that could potentially influence stroke outcome. In the acute phase of stroke, particular forms of neuronal activity may modulate vascular flow leading to neuronal survival and improved outcome. ${ }^{48,49}$ By contrast, other forms of activity may increase metabolic load and exacerbate damage through peri-infarct depolarizations..$^{23,43,50}$ The role of activity in the acute stroke setting (first hours to weeks) is critical for not only saving tissue but also defining the time window where activity-based interventions such as rehabilitation can be safely initiated. Recent work in rehabilitation has shown that earlier mobilization after stroke leads to poor outcome. ${ }^{51}$ Integrating basic and clinical research results is also necessary to develop knowledge that can lead to the development of translational rehabilitation aids and implement brain-machine interfaces or closed-loop stimulation to restore function in patients. ${ }^{52,53}$ We suggest that chronic stroke models that lack the confounding effects of anesthesia offer the ideal scenario to manipulate activity at short-time windows following stroke induction or to best stratify animals into groups with similar lesions where later manipulations can be tested with lower variance.

\section{Disclosures}

No competing financial interests exist.

\section{Acknowledgments}

This work was supported by the Leducq Foundation and a Foundation Grant from the Canadian Institutes of Health Research FDN-143209, and the Brain Canada, Canadian Neurophotonics Platform. It was also supported by a grant from Aid from the Heart and Stroke Foundation of Canada. We thank Pumin Wang for surgical assistance.

\section{References}

1. C. J. Murray and A. D. Lopez, "Global mortality, disability, and the contribution of risk factors: global burden of disease study," Lancet 349, 1436-1442 (1997).

2. H. M. Dewey et al., "Cost of stroke in Australia from a societal perspective: results from the North East Melbourne Stroke Incidence Study (NEMESIS)," Stroke 32, 2409-2416 (2001).

3. E. C. Jauch et al., "Guidelines for the early management of patients with acute ischemic stroke: a guideline for healthcare professionals from the American Heart Association/American Stroke Association," Stroke 44, 870-947 (2013).

4. V. E. O'Collins et al., "1,026 experimental treatments in acute stroke," Ann. Neurol. 59, 467-477 (2006).

5. M. D. Hill et al., "Safety and efficacy of NA-1 in patients with iatrogenic stroke after endovascular aneurysm repair (ENACT): a phase 2, randomised, double-blind, placebo-controlled trial," Lancet Neurol. 11, 942-950 (2012).

6. T. Yamakura and R. A. Harris, "Effects of gaseous anesthetics nitrous oxide and xenon on ligand-gated ion channels. Comparison with isoflurane and ethanol," Anesthesiology 93, 1095-1101 (2000).

7. N. Kotani and N. Akaike, "The effects of volatile anesthetics on synaptic and extrasynaptic GABA-induced neurotransmission," Brain Res. Bull. 93, 69-79 (2013).

8. S. Strebel et al., "Dynamic and static cerebral autoregulation during isoflurane, desflurane, and propofol anesthesia," Anesthesiology 83, 66-76 (1995).

9. A. Seto et al., "Induction of ischemic stroke in awake freely moving mice reveals that isoflurane anesthesia can mask the benefits of a neuroprotection therapy," Front. Neuroenerg. 6, 1 (2014).

10. P. J. Drew et al., "Chronic optical access through a polished and reinforced thinned skull," Nat. Methods 7, 981-984 (2010).

11. G. Silasi et al., "Intact skull chronic windows for mesoscopic wide-field imaging in awake mice," J. Neurosci. Methods 267, 141-149 (2016).

12. M. P. Vanni and T. H. Murphy, "Mesoscale transcranial spontaneous activity mapping in GCaMP3 transgenic mice reveals extensive reciprocal connections between areas of somatomotor cortex," J. Neurosci. 34, 15931 (2014).

13. A. Sigler, A. Goroshkov, and T. H. Murphy, "Hardware and methodology for targeting single brain arterioles for photothrombotic stroke on an upright microscope," J. Neurosci. Methods 170, 35-44 (2008).

14. B. D. Watson et al., "Induction of reproducible brain infarction by photochemically initiated thrombosis," Ann. Neurol. 17, 497-504 (1985).

15. A. K. Dunn et al., "Dynamic imaging of cerebral blood flow using laser speckle," J. Cereb. Blood Flow Metab. 21, 195-201 (2001).

16. L. Madisen et al., "A toolbox of Cre-dependent optogenetic transgenic mice for light-induced activation and silencing," Nat. Neurosci. 15, 793-802 (2012).

17. www.well.ca

18. R. D. Frostig et al., "Cortical functional architecture and local coupling between neuronal activity and the microcirculation revealed by in vivo high-resolution optical imaging of intrinsic signals," Proc. Natl. Acad. Sci. U. S. A. 87, 6082-6086 (1990).

19. Allen Brain Atlas Data Portal, http://brain-map.org

20. T. C. Harrison et al., "Displacement of sensory maps and disorganization of motor cortex after targeted stroke in mice," Stroke 44, 23002306 (2013).

21. C. B. Schaffer et al., "Two-photon imaging of cortical surface microvessels reveals a robust redistribution in blood flow after vascular occlusion," PLoS Biol. 4, e22 (2006).

22. S. M. Kazmi et al., "Chronic imaging of cortical blood flow using multiexposure speckle imaging," J. Cereb. Blood Flow Metab. 33, 798-808 (2013).

23. G. C. Petzold et al., "Increased extracellular $\mathrm{K}^{+}$concentration reduces the efficacy of N-methyl-D-aspartate receptor antagonists to block spreading depression-like depolarizations and spreading ischemia," Stroke 36, 1270-1277 (2005).

24. A. B. Steffensen et al., "Chloride cotransporters as a molecular mechanism underlying spreading depolarization-induced dendritic beading," J. Neurosci. 35, 12172 (2015).

25. T. H. Murphy et al., "Two-photon imaging of stroke onset in vivo reveals that NMDA-receptor independent ischemic depolarization is 
the major cause of rapid reversible damage to dendrites and spines," J. Neurosci. 28, 1756-1772 (2008).

26. E. Busch et al., "Simultaneous recording of EEG, DC potential and diffusion-weighted NMR imaging during potassium induced cortical spreading depression in rats," NMR Biomed. 8, 59-64 (1995).

27. G. Yang et al., "Human copper-zinc superoxide dismutase transgenic mice are highly resistant to reperfusion injury after focal cerebral ischemia," Stroke 25, 165-170 (1994).

28. V. Labat-Gest and S. Tomasi, "Photothrombotic ischemia: a minimally invasive and reproducible photochemical cortical lesion model for mouse stroke studies," J. Vis. Exp. (76), e50370 (2013).

29. C. Ayata and M. A. Moskowitz, "Cortical spreading depression confounds concentration-dependent pial arteriolar dilation during N-methyl-D-aspartate superfusion," Am. J. Physiol. Heart Circ. Physiol. 290, H1837 (2006).

30. S. Chen et al., "Time-varying spreading depression waves in rat cortex revealed by optical intrinsic signal imaging," Neurosci. Lett. 396, 132-136 (2006).

31. W. I. Rosenblum and F. El-Sabban, "Platelet aggregation in the cerebral microcirculation: effect of aspirin and other agents," Circ. Res. 40, 320-328 (1977).

32. A. W. Gelb et al., "Propofol anesthesia compared to awake reduces infarct size in rats," Anesthesiology 96, 1183-1190 (2002).

33. G. Yang et al., "Thinned-skull cranial window technique for long-term imaging of the cortex in live mice," Nat. Protoc. 5, 201-208 (2010).

34. P. J. Helm, O. P. Ottersen, and G. Nase, "Analysis of optical properties of the mouse cranium-implications for in vivo multi photon laser scanning microscopy," J. Neurosci. Methods 178, 316-322 (2009).

35. A. M. Valentim et al., "The effects of depth of isoflurane anesthesia on the performance of mice in a simple spatial learning task," J. Am. Assoc. Lab. Anim. Sci. 47(3), 16-19 (2008).

36. C. Constantinides, R. Mean, and B. J. Janssen, "Effects of isoflurane anesthesia on the cardiovascular function of the C57BL/6 mouse," ILAR J. 52(3), e21-e31 (2011).

37. W. Erhardt et al., "A comparative study with various anesthetics in mice (pentobarbitone, ketamine-xylazine, carfentanyl-etomidate)," Res. Exp. Med. 184, 159-169 (1984).

38. D. H. Lim et al., "Optogenetic mapping after stroke reveals networkwide scaling of functional connections and heterogeneous recovery of the peri-infarct," J. Neurosci. 34, 16455 (2014).

39. A. Y. Shih et al., "The smallest stroke: occlusion of one penetrating vessel leads to infarction and a cognitive deficit," Nat. Neurosci. 16, $55-63(2013)$.
40. N. Nishimura et al., "Targeted insult to subsurface cortical blood vessels using ultrashort laser pulses: three models of stroke," Nat. Methods $\mathbf{3}$, 99-108 (2006).

41. J. M. Jia et al., "Control of cerebral ischemia with magnetic nanoparticles," Nat. Methods 14, 160-166 (2016).

42. D. A. Dombeck et al., "Imaging large-scale neural activity with cellular resolution in awake, mobile mice," Neuron 56(1), 43-57 (2007).

43. W. C. Risher et al., "Recurrent spontaneous spreading depolarizations facilitate acute dendritic injury in the ischemic penumbra," J. Neurosci. 30, 9859-9868 (2010).

44. J. Mantz, J. Cordier, and C. Giaume, "Effects of general anesthetics on intercellular communications mediated by gap junctions between astrocytes in primary culture," Anesthesiology 78, 892-901 (1993).

45. S. T. Carmichael, "Rodent models of focal stroke: size, mechanism, and purpose," NeuroRX 2, 396-409 (2005).

46. F. Li, T. Omae, and M. Fisher, "Spontaneous hyperthermia and its mechanism in the intraluminal suture middle cerebral artery occlusion model of rats," Stroke 30, 2464-2471 (1999).

47. K. Tureyen et al., "Ideal suture diameter is critical for consistent middle cerebral artery occlusion in mice," Neurosurgery 56, 382-390 (2005).

48. M. Y. Cheng et al., "Optogenetic neuronal stimulation promotes functional recovery after stroke," Proc. Natl. Acad. Sci. U. S. A. 111, 12913 (2014).

49. C. C. Lay and R. D. Frostig, "Complete protection from impending stroke following permanent middle cerebral artery occlusion in awake, behaving rats," Eur. J. Neurosci. 40, 3413-3421 (2014).

50. D. von Bornstadt et al., "Supply-demand mismatch transients in susceptible peri-infarct hot zones explain the origins of spreading injury depolarizations," Neuron 85, 1117-1131 (2015).

51. J. Bernhardt et al., "Efficacy and safety of very early mobilisation within $24 \mathrm{~h}$ of stroke onset (AVERT): a randomised controlled trial," Lancet 386, 1734-1735 (2015)

52. D. J. Guggenmos et al., "Restoration of function after brain damage using a neural prosthesis," Proc. Natl. Acad. Sci. U. S. A. 110, 21177 (2013).

53. T. Gulati et al., "Robust neuroprosthetic control from the stroke perilesional cortex," J. Neurosci. 35, 8653-8661 (2015).

Biographies for the authors are not available. 